\title{
Waves generated by reflected and accelerated electrons at a nearly perpendicular shock
}

\author{
M. Karlický and M. Vandas \\ Astronomical Institute of the Academy of Sciences of the Czech Republic, 25165 Ondřejov, Czech Republic \\ e-mail: karlicky@asu.cas.cz \\ Received 22 November 2005 / Accepted 17 March 2006 \\ ABSTRACT

\begin{abstract}
Aims. We study a generation of waves by reflected and accelerated electrons at a nearly perpendicular shock.
Methods. A shifted loss-cone distribution of reflected and accelerated electrons at a nearly perpendicular shock is derived analytically. Then in a simplified form called here "cone" beam, this distribution is incorporated into a 2D relativistic electromagnetic particle-incell model and the wave generation is studied.

Results. Numerical modelling shows not only a generation of Langmuir and high-frequency electromagnetic waves, as expected, but also an efficient generation of whistler waves by the normal Doppler resonance process. While the intensity of Langmuir waves increases with the increase in the "cone" beam energy in a parallel direction to the magnetic field, the whistlers are most effectively generated for high values of the perpendicular beam energy. There are indications that the whistlers participate in conversion processes generating the high-frequency electromagnetic waves.

Conclusions. Using a numerical model we found an efficient generation of Langmuir, whistler, and electromagnetic waves by electrons reflected and accelerated at a nearly perpendicular shock. Results are discussed in connection with the herringbone structure of
\end{abstract} \\ type II radio bursts. The possible role of transmitted electrons is mentioned.
}

Key words. Shock waves - Sun: radio radiation

\section{Introduction}

It is commonly accepted that nearly perpendicular shocks accelerate electrons (Holman \& Pesses 1983; Wu 1984; Leroy \& Mangeney 1984; Vandas 1989a,b; Mann \& Klassen 2005) and that these electrons generate type II solar radio bursts (Nelson \& Melrose 1985). Observations of interplanetary type II radio bursts made by Bale et al. (1999) support this concept.

The recent theory of type II radio bursts is presented in the papers by Knocks et al. (2001, 2003). They propose shockdrift acceleration of electrons at quasi-perpendicular shock, they compute the integrated (along the velocity component perpendicular to the magnetic field) distribution function of electrons reflected, and they show a formation of the bump-on-tail distribution function in a foreshock space. Then in considering the bump-on-tail instability, they compute a generation of Langmuir waves and their conversion to electromagnetic mode. They assume the decay process $L \rightarrow T+S$ ( $L$ denotes a beam-driven Langmuir wave, $S$ denotes an ion-sound wave and $T$ denotes a transverse wave) for the emission on the fundamental frequency and the coalescence process $L+L^{\prime} \rightarrow T$ ( $L^{\prime}$ denotes the secondary Langmuir wave produced in the Langmuir wave decay) for the harmonic emission.

A similar model, but for herringbone structures of the type II solar radio bursts (Roberts 1959), has been proposed by Mann $\&$ Klassen (2005). The herringbone structure resembles a group of type III bursts, but with the frequency drift, which is about one half of that of type III bursts (Zaitsev et al. 1998; Mann \& Klassen 2002).
In the present paper we start from the same concept as Knocks et al. (2001, 2003) and Mann \& Klassen (2005), but we consider the full (not integrated in velocity space) distribution function of reflected electrons. In a simplified form we incorporated this full distribution function into a $2 \mathrm{D}$ relativistic electromagnetic particle-in-cell model, and found an efficient production of whistlers that have not been considered in previous studies and that modify the processes generating high-frequency electromagnetic waves (radio radiation).

\section{Distribution function of electrons reflected at a nearly perpendicular shock}

The theory of acceleration and reflection of suprathermal electrons at a nearly perpendicular plane shock wave have been well-established (Holman \& Pesses 1983; Wu 1984; Leroy \& Mangeney 1984; Vandas 1989a,b). A nearly perpendicular shock wave acts as a fast moving magnetic mirror with the effective magnetic mirror velocity

$V_{B}=V_{1 n} / \cos \theta_{B n}$

and the loss cone angle

$\theta_{\mathrm{c}}=\arcsin \sqrt{1 / v}$

where $V_{1 n}$ is the normal component of the upstream plasma velocity to the shock, $\theta_{B n}$ is the angle between the upstream magnetic field vector and the shock normal, and $v$ is the magnetic field jump at the shock (the ratio of downstream to upstream magnetic field magnitude). This angle $\theta_{B n}$ must be close 
to $90^{\circ}$ (within several degrees) for reflection to be effective. Reflected electrons propagate upstream away from a shock and form a beam with a loss cone.

The initial velocity $v_{\mathrm{i}}$ and pitch angle $\alpha_{\mathrm{i}}$ are related to the final velocity $v_{\mathrm{f}}$ and pitch angle $\alpha_{\mathrm{f}}$ (after reflection) by the equations (e.g., Vandas 1989a)

$v_{\mathrm{i}}^{2}=v_{\mathrm{f}}^{2}+4 V_{B} v_{\mathrm{f}} \cos \alpha_{\mathrm{f}}+4 V_{B}^{2}$,

$v_{\mathrm{i}} \cos \alpha_{\mathrm{i}}=-v_{\mathrm{f}} \cos \alpha_{\mathrm{f}}-2 V_{B}$.

The velocities and pitch angles are in plasma rest frame, and electrons moving toward the shock (in the upstream region) have $\alpha<90^{\circ}$ (therefore reflected electrons will have $\alpha_{\mathrm{f}}>90^{\circ}$ ). Note that according to our definition, $\alpha$ would not change if we changed the upstream magnetic field $\boldsymbol{B}_{1}$ to $-\boldsymbol{B}_{1}$ (because the acceleration and reflection are not sensitive to the direction of $\boldsymbol{B}_{1}$ ). The final velocity $v_{\mathrm{f}}$ and pitch angle $\alpha_{\mathrm{f}}$ fulfill (Vandas 1989a)

$v_{\mathrm{f}}>V_{B}, \quad \alpha_{\mathrm{f} k}<\alpha_{\mathrm{f}}<\alpha_{\mathrm{f} c}$,

where

$\cos \alpha_{\mathrm{f} k}=-\frac{V_{B}}{v_{\mathrm{f}}}$,

$\cos \alpha_{\mathrm{f} c}=-\frac{V_{B} \sin ^{2} \theta_{\mathrm{c}}+\cos \theta_{\mathrm{c}} \sqrt{v_{\mathrm{f}}^{2}-V_{B}^{2} \sin ^{2} \theta_{\mathrm{c}}}}{v_{\mathrm{f}}}$.

Using the expressions (1)-(7) and the Liouville theorem, a distribution function of reflected electrons can be constructed from an initial distribution function.

Vandas \& Karlický (2000) examined acceleration of electrons by a coronal shock. We shall use the same parameters for the electron initial distribution function and the shock as they did. It is assumed that the initial distribution function consists of thermal electrons (core, C) and a suprathermal tail (halo, $\mathrm{H}$ )

$f_{\mathrm{i}}(v, \alpha)=f_{\mathrm{C}}(v)+f_{\mathrm{H}}(v)$,

with a Maxwellian core

$f_{\mathrm{C}}(v)=\frac{n_{\mathrm{C}}}{\pi^{3 / 2} v_{\mathrm{C}}^{3}} \exp \left(-\frac{v^{2}}{v_{\mathrm{C}}^{2}}\right)$

and halo electrons approximated by a kappa distribution function

$f_{\mathrm{H}}(v)=\frac{n_{\mathrm{H}} c_{\kappa}}{\pi^{3 / 2} v_{\mathrm{H}}^{3}}\left[1+\frac{v^{2}}{v_{\mathrm{H}}^{2}\left(\kappa-\frac{3}{2}\right)}\right]^{-\kappa-1}$,

where $n_{\mathrm{C}}$ is the density of thermal electrons, $v_{\mathrm{C}}$ their thermal speed, $n_{\mathrm{H}}$ the halo density, and $v_{\mathrm{H}}$ is the characteristic halo electron velocity,

$c_{\kappa}=\frac{\Gamma(\kappa+1)}{\Gamma\left(\kappa-\frac{1}{2}\right)\left(\kappa-\frac{3}{2}\right)^{3 / 2}}$.

Here $\Gamma$ is the gamma function, and $\kappa$ determines the deviation of the halo distribution from a Maxwellian one.

A contribution of core electrons to the flux of reflected electrons is negligible in the keV-range (Vandas 1989a) so it is neglected. Therefore a distribution function of reflected electrons will be

$f_{\mathrm{R}}(v, \alpha)=\frac{n_{\mathrm{H}} c_{\kappa}}{\pi^{3 / 2} v_{\mathrm{H}}^{3}}\left[1+\frac{v^{2}+4 V_{B} v \cos \alpha+4 V_{B}^{2}}{v_{\mathrm{H}}^{2}\left(\kappa-\frac{3}{2}\right)}\right]^{-\kappa-1}$,

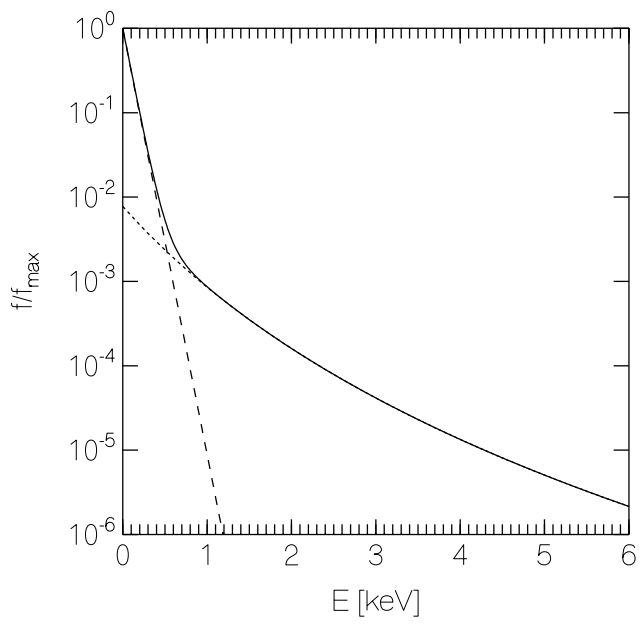

Fig. 1. The initial distribution function of coronal electrons (solid line) consisting of a core (dashed line) and a halo (dotted line).

which is valid for

$v>V_{B}, \quad \alpha_{\mathrm{f} k}<\alpha<\alpha_{\mathrm{f} c}$,

otherwise $f_{\mathrm{R}}=0$.

In an analogy to in-situ solar wind measurements we set $\frac{1}{2} m v_{\mathrm{H}}^{2}=0.6 \mathrm{keV}$ ( $m$ is the electron mass), $n_{\mathrm{H}} / n_{\mathrm{C}}=0.1$, and $\kappa=6$ (Vandas \& Karlický 2000). It is $\frac{1}{2} m v_{\mathrm{C}}^{2} \approx 86 \mathrm{eV}$ for a coronal temperature of $10^{6} \mathrm{~K}$. A coronal shock has the following parameters: the upstream magnetic field $B_{1}=0.5 \mathrm{mT}(5 \mathrm{G})$, the velocity $V_{1 n}=1000 \mathrm{~km} \mathrm{~s}^{-1}$, the angle $\theta_{B n}=86^{\circ}$, and the magnetic field jump $v=1.6$.

The initial distribution function (8) is shown in Fig. 1. An angular distribution function of reflected electrons (12) is shown in Fig. 2 for the energy of $5 \mathrm{keV}$. For comparison, using the same mathematical tools, the distribution function of the transmitted electrons in the nearly-perpendicular shock is added (bottom part of Fig. 2).

\section{Numerical model}

To simulate the wave processes connected with the reflected electrons in the nearly perpendicular shock, we use a $2 \mathrm{D} 3 \mathrm{~V}$ (2 spatial and 3 velocity components) fully relativistic electromagnetic particle-in-cell code (Saito \& Sakai 2004). The system size is $L_{x}=512 \Delta$ and $L_{y}=6 \Delta$, where $\Delta(=1)$ is a grid size. In each cell 100 numerical electrons and 100 numerical protons are initiated. The periodic boundary conditions for both $x$ - and $y$-directions are imposed on particles and electromagnetic fields. We consider electron-proton plasma in the magnetic field $(\boldsymbol{B} \equiv$ $\left.\left(\mathrm{B}_{x}, 0,0\right)\right)$ with the real proton-electron mass ratio $m_{\mathrm{p}} / m_{\mathrm{e}}=1836$.

For all computations presented here, temperatures of the numerical background electrons and protons are chosen to be equal to the coronal temperature $\left(T_{\mathrm{e}}=T_{\mathrm{p}}=10^{6} \mathrm{~K}\right)$, i.e., the electron thermal velocity is $v_{T \mathrm{e}}=0.0129 c$, where $c$ is the speed of light.

As shown in the previous analysis the distribution function of reflected electrons has the form of a so-called shifted losscone distribution. To model it in a simplified form, we consider a cloud of numerical electrons which have the distribution ("cone" beam for short) as follows: the mean velocity parallel to the magnetic field $v_{x}^{b}=\left|v^{b}\right| \cos \alpha^{b}$, the mean velocity perpendicular to the magnetic field $v_{\text {perp }}^{b}=\left|v^{b}\right| \sin \alpha^{b}$, and the beam thermal velocity $v_{T \mathrm{e}}^{b}=0.0129 c$, where $\alpha^{b}$ is the angle between the 


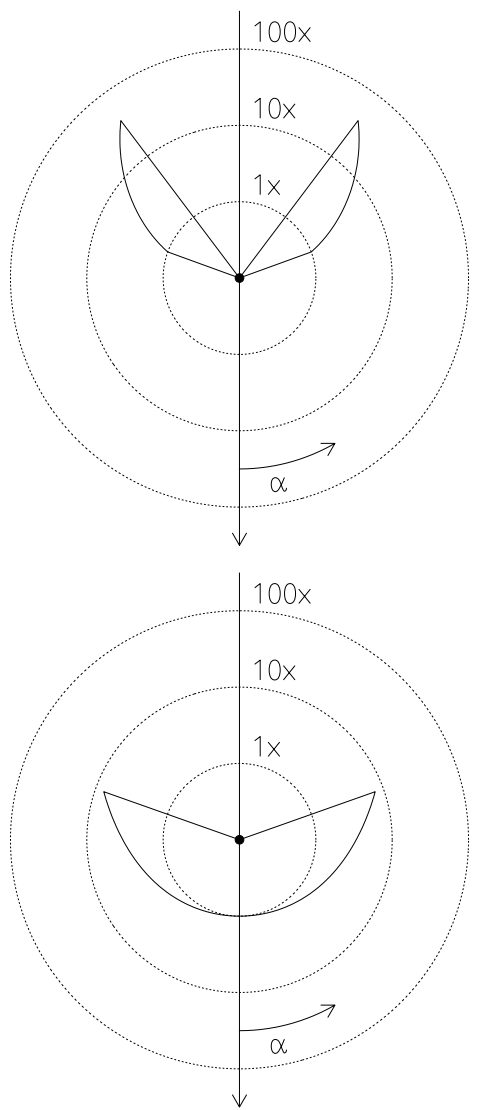

Fig. 2. Upper: angular distribution function of reflected electrons for the energy of $5 \mathrm{keV}$. The polar graph shows a dependence of $\log f_{\mathrm{R}}(v, \alpha) / f_{\mathrm{i}}(v, \alpha)$ on $\alpha$ for a fixed $v$. Because $f_{\mathrm{i}}$ does not depend on $\alpha$, all $f_{\mathrm{R}}$ values are divided by a constant value given by the inner dotted circle and labelled $1 \times$. The other circles indicate values $10 \times$ and $100 \times$ higher than $f_{\mathrm{i}}$. The vertical line is a direction of the upstream magnetic field $\boldsymbol{B}_{1}$ and the arrow indicates a direction to the shock. The arc with an arrow shows how the pitch angle $\alpha$ is counted from $0^{\circ}$ to $180^{\circ}$. The distribution function is rotationally symmetric around the vertical line. Bottom: angular distribution function of transmitted electrons for the energy of $5 \mathrm{keV}$. The vertical line is a direction of the downstream magnetic field and the arrow indicates a direction away from the shock.

"cone" beam velocity $\boldsymbol{v}^{b}$ and the magnetic field $\boldsymbol{B}$. An example of the distribution function of the "cone" beam is shown in Fig. 3.

\subsection{Results}

Due to limitations of the numerical model, the values of the beam density and magnetic field in the model are chosen higher than those in real coronal conditions. The model parameters are that the density ratio between beam and background plasma is $n^{b} / n_{\mathrm{e}}=0.05$ and the ratio between electron cyclotron and plasma frequencies are $\omega_{B \mathrm{e}} / \omega_{\mathrm{pe}}=0.5$. The mean total beam velocity is chosen $\left|v^{b}\right|=0.44 c(50 \mathrm{keV})$; compare with the energy of the beams generating the herringbone structure $(0.2-80 \mathrm{keV}$, see Mann \& Klassen 2005).

Computations with three different angles $\alpha^{b}=30^{\circ}, 45^{\circ}$, and $60^{\circ}$ were made in the time interval $0-500 \omega_{\text {pe }} t$. Results of computations are summarized in Figs. 4-8, while Fig. 4 shows an example of the time evolution of the electric field energy (electrostatic $x$-component) for the case with the angle $\alpha^{b}=45^{\circ}$. During the process the distribution of electrons evolves to what
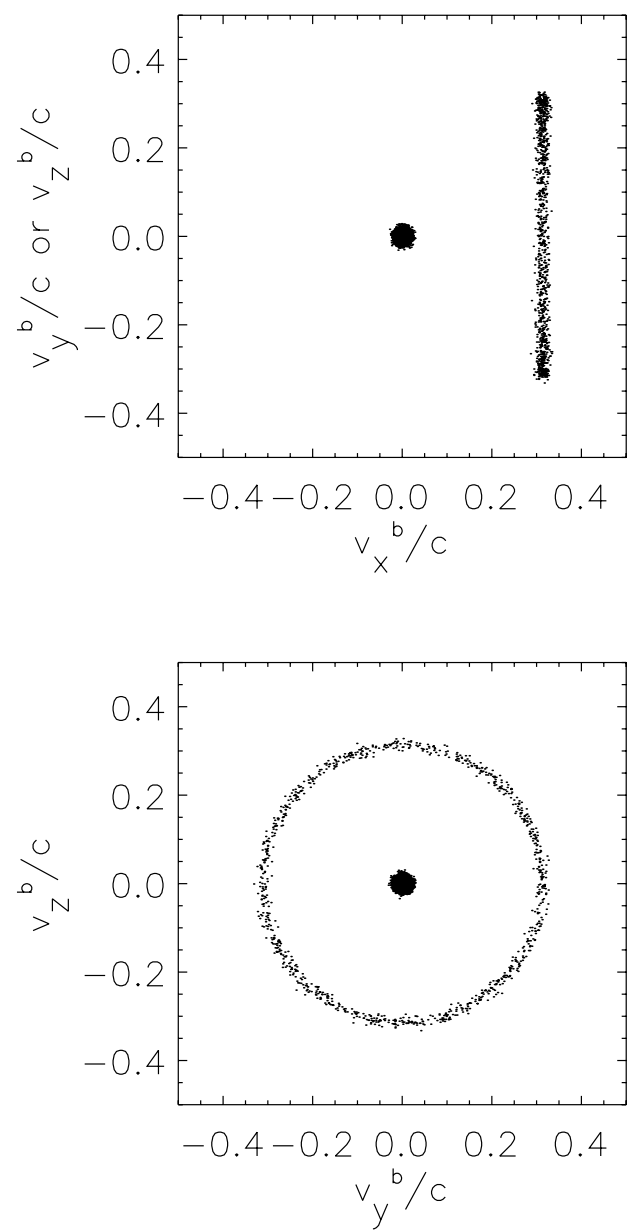

Fig. 3. The initial electron distribution of the background plasma and "cone" beam for the case with the angle $\alpha^{b}=45^{\circ}$, where $c$ means the speed of light.

is shown in Fig. 5. This type of electron distribution is a typical one for all computations up to $500 \omega_{\mathrm{pe}} t$.

Then for all computations, the dispersion diagrams for the electric field components $E_{x}$ and $E_{z}$, measured along the $x$-coordinate $(y=3 \Delta)$ for the time interval $\left(0-200 \omega_{\text {pe }} t\right)$, were constructed using the 2D Fourier transform. Examples of these dispersion diagrams are presented in Figs. 6 and 7. The maxima of Langmuir, whistler, left-hand, and right-hand polarized highfrequency electromagnetic waves can be found inside boxes 1-4.

As can be seen here the beam strongly generates Langmuir waves by the two-stream instability for the resonant condition $\omega=k_{x} v_{x}^{b}$ (Čerenkov type), see the emission in the boxes numbered 1 in Figs. 6 and 7 (left parts). Their real frequency is lower than the plasma frequency of the background plasma $\omega_{\text {pe }}$, and the difference corresponds to the analytical estimation (Mikhailovskii 1974):

$\Delta \omega=-\omega_{\mathrm{pe}} \alpha^{1 / 3} / 2^{4 / 3}=-0.15 \omega_{\mathrm{pe}}$,

where $\alpha$ is the ratio of the beam and plasma densities $(0.05$ in our case). In a short time backscattered Langmuir waves appeared in a very broad range of $k$ at the frequency $\omega \approx \omega_{\text {pe }}$. The beam generates the whistler waves (see the emission in the boxes numbered 2 in Figs. 6 and 7), too. It is interesting to see that the whistler waves have a maximum for those propagating backwards compared to the beam propagation. Detailed analysis of this fact shows that the case under study resembles the 


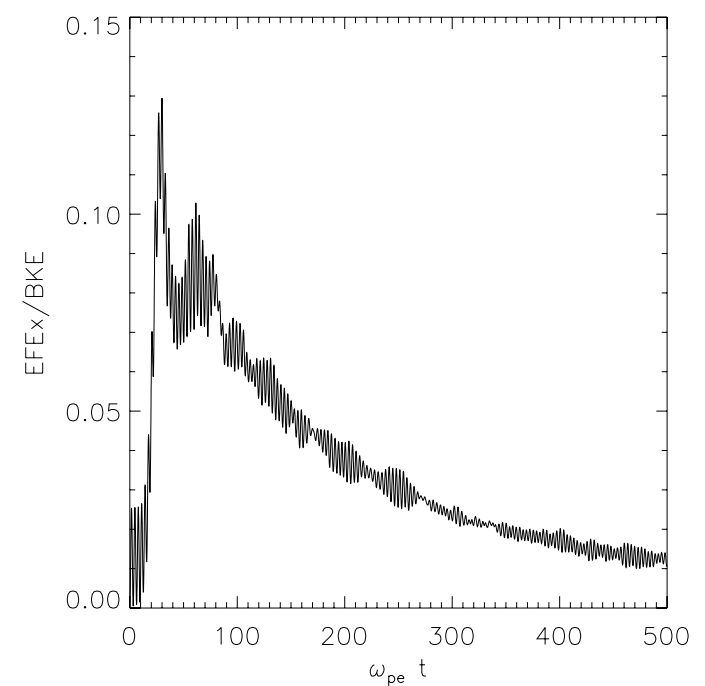

Fig. 4. The evolution of the electric field energy ( $x$-component) EFEx expressed in the ratio to the beam kinetic energy BKE for the case with the angle $\alpha^{b}=45^{\circ}$.

case of the electromagnetic instability of the beam with some finite energy in the perpendicular direction to the magnetic field (Mikhailovskii 1974). Similar to in this case, we see a generation of whistler waves (w) by the normal Doppler resonance process (Melrose 1980) expressed by the condition:

$\omega_{\mathrm{w}}-\omega_{B e}-k_{x} v_{x}^{b} \approx 0$.

Because the frequency of the whistler waves is lower than the electron cyclotron frequency $\omega_{w}<\omega_{B e}$, the resonant $k$-vector is negative

$k_{x} \approx-\left(\omega_{B e}-\omega_{w}\right) / v_{x}^{b}$,

i.e., oriented in the opposite direction compared to beam propagation.

Furthermore, the dispersion diagrams for $E_{z}$ computed along the $x$-axis (right parts of Figs. 6 and 7) show dispersion curves of the left-hand and right-hand polarized high-frequency electromagnetic waves. We found their maxima at places inside the boxes 3 and 4 in Figs. 6 and 7. To understand better these processes we made an inverse 2D Fourier transform of intensities in the $k-\omega$ space in the boxes 1, 2, 3, and 4 for all three considered beam angles. Resulting temporal evolutions of Langmuir, whistler, and electromagnetic waves are shown in Fig. 8.

As seen, the most intense waves are Langmuir waves and their maxima decrease with the increase in the beam angle $\alpha^{b}$, i.e. with the decrease in $v_{x}^{b}$ for constant $\left|v^{b}\right|$. The growth rate of the Langmuir waves is $\gamma_{L}=0.18 \omega_{\text {pe }}$, which is lower than the maximum growth rate estimated by Mikhailovskii (1974) for the monoenergetic beam:

$\frac{\gamma_{L}}{\omega_{\mathrm{pe}}}=\frac{\sqrt{3}}{2^{4 / 3}}\left(\frac{n_{b}}{n_{e}}\right)^{1 / 3}=0.25$.

On the other hand, the whistler waves are the most efficiently generated for the beam angle $\alpha^{b}=60^{\circ}$, where the growth rate is $\gamma_{\mathrm{W}}=0.013 \omega_{\mathrm{pe}}$. As far as the electromagnetic waves are concerned, their maxima production is also found for higher beam angles. It indicates the relationship between the production of electromagnetic waves and energy levels of whistler waves. Similar results were also found for computations with
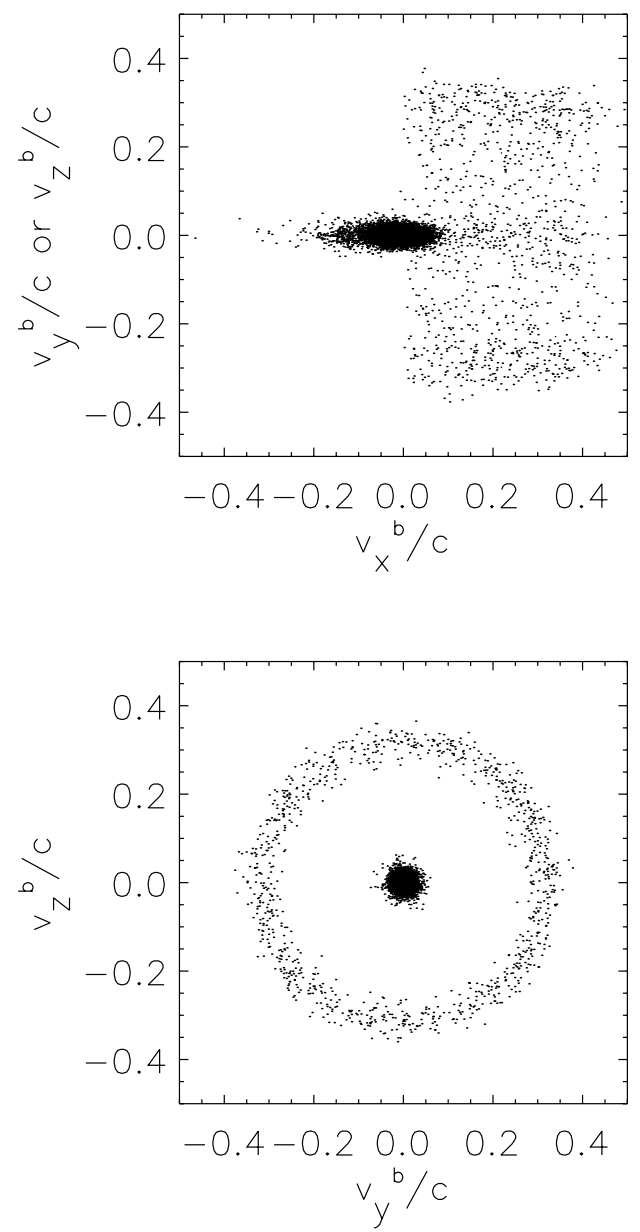

Fig. 5. The electron distribution of the background plasma and "cone" beam at $\omega_{\mathrm{pe}} t=200$ for the case with the angle $\alpha^{b}=45^{\circ}$, where $c$ means the speed of light.

the lower ratio of electron cyclotron and plasma frequencies, for $\omega_{B e} / \omega_{\mathrm{pe}}=0.3$, and for unequal temperatures of background plasma electrons and protons, $T_{\mathrm{e}}=5 \times T_{\mathrm{p}}$ with $T_{\mathrm{p}}=10^{6} \mathrm{~K}$.

\section{Discussion and conclusions}

Using the 2D relativistic electromagnetic code for a system of plasma with the "cone" beam, representing the shifted losscone distribution of reflected electrons at a nearly perpendicular shock, we found efficient generation of Langmuir, whistler, and high-frequency electromagnetic waves. We considered a distribution function without an integration along the velocity component perpendicular to the magnetic field as made in previous studies (Knocks et al. 2001, 2003; Mann \& Klassen 2005). This change leads to generation of whistlers. Just the whistler waves, which are produced by the normal Doppler resonance process in opposite direction to the "cone" beam propagation, are a new aspect of these processes. This result can be supported by observations of whistler waves in connection with reflected electrons upstream of the Earth's bow shock (Trávníček et al. 2002).

Our simulations show that, while Langmuir waves are most effectively generated by beams that are nearly parallel to the magnetic field, the level of the energy of the whistlers increases with the beam energy perpendicular to the magnetic field. The maxima production of the electromagnetic waves is also found for higher beam angles. It indicates the relationship between the 

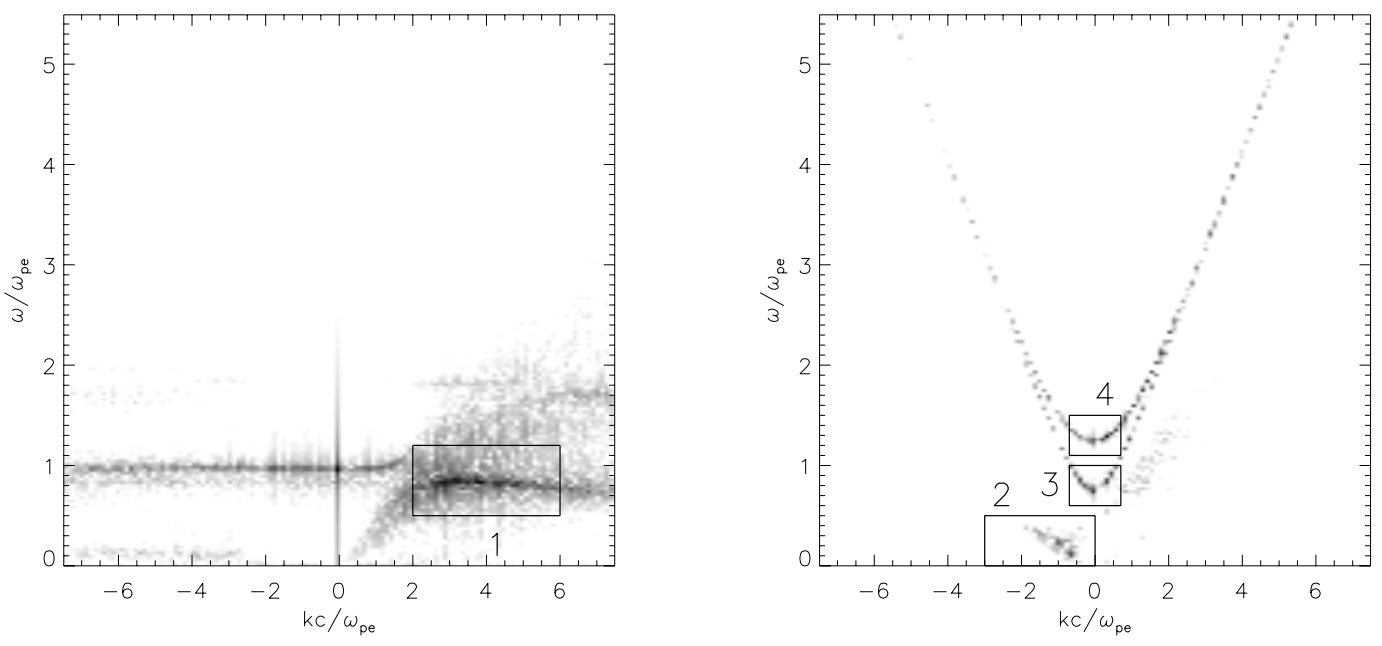

Fig. 6. Dispersion diagrams for the case with the angle $\alpha^{b}=30^{\circ}$. Left: the diagram for the electric field $E_{x}$ parallel to the magnetic field. Right: the diagram for the electric field $E_{z}$ perpendicular to the magnetic field. The boxes designated as 1, 2, 3, and 4 show the $k$ - $\omega$ spaces with the maxima of Langmuir, whistler, left-hand, and right-hand polarized electromagnetic waves, respectively, which were used for the computations of their temporal evolutions presented in Fig. 8.
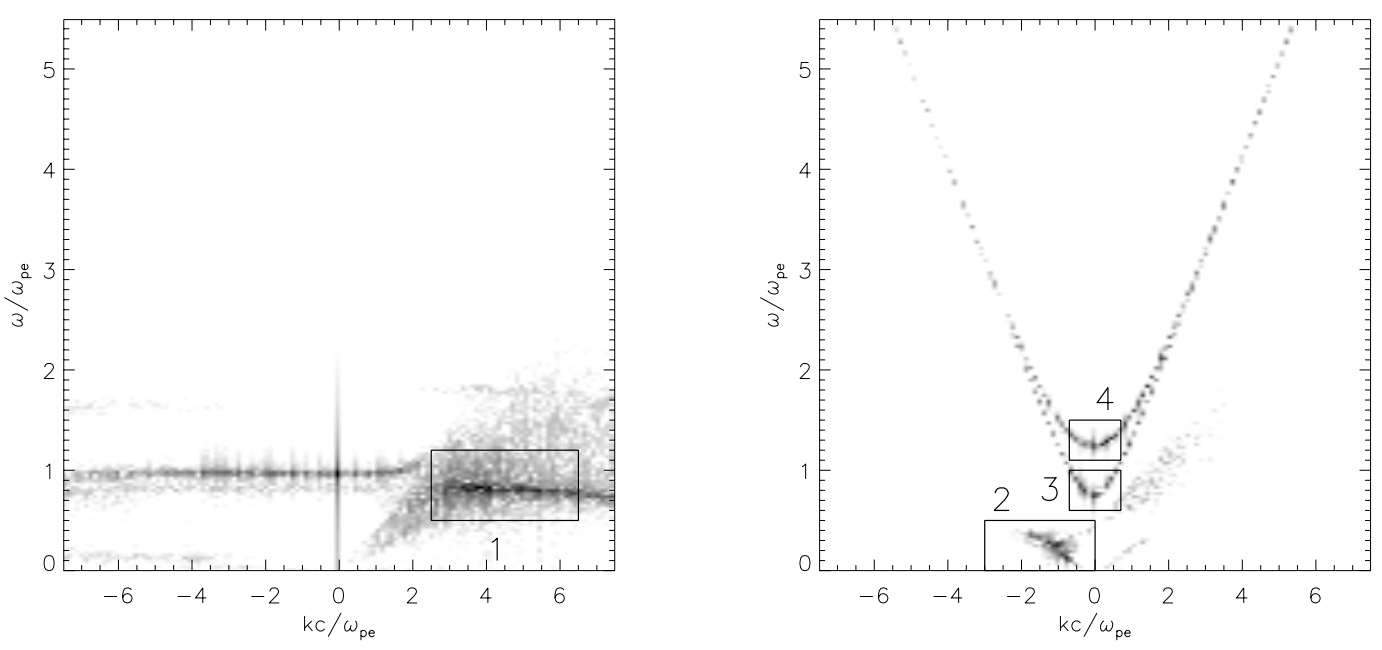

Fig. 7. Dispersion diagrams for the case with the angle $\alpha^{b}=45^{\circ}$. Format is the same as in Fig. 6 .

production of electromagnetic waves and the energy level of the whistler waves.

For an explanation of the influence of whistlers on electromagnetic modes, we propose the following wave-wave processes.

a) The right-hand polarized electromagnetic waves (box 4 in Figs. 6 and 7):

Probably we see the coalescence process $\mathrm{L}+\mathrm{W} \rightarrow \mathrm{T}$ here in which the Langmuir (L) and whistler (W) plasmons form the electromagnetic one (T) (Tsytovich 1970). In this process the energy and momentum conservation laws $\left(\omega_{\mathrm{L}}+\omega_{\mathrm{W}}=\omega_{\mathrm{T}}\right.$ and $k_{\mathrm{L}}+k_{\mathrm{W}}=k_{\mathrm{T}}$ ) have to be fulfilled. Looking to the dispersion diagrams (Fig. 6 and 7), such a process is possible because for the maxima of whistler waves in boxes numbered 2 it is possible to find $k$ and $\omega$ of the Langmuir waves, which using the conservation laws give the maxima in the boxes 4 for the electromagnetic mode.

b) The left-hand polarized electromagnetic waves (box 3 in Figs. 6 and 7):

In this case the decay process with the ion-sound waves $L \rightarrow T+S$, proposed by Knocks et al. (2001), is possible, due especially to the fact that the process generating backscattered Langmuir waves produces the ion-sound waves (which are strongly damped for the $T_{\mathrm{e}}=T_{\mathrm{p}}$ case) (Bárta \& Karlický 2000). Note that in the present model with a limited frequency resolution the ion-sound waves cannot be identified.

On the other hand, due to the increase in the energies of the whistler and electromagnetic waves found for high values of the "cone" beam angle, we think that the decay process of the Langmuir plasmon into the left-hand electromagnetic and whistler plasmons $\mathrm{L} \rightarrow \mathrm{T}+\mathrm{W}$, induced by a relatively high energy level of the whistlers, is also possible (see also Tsytovich 1970); namely, for this process we can find the Langmuir waves fitting the conservation laws.

Our simulations correspond to early phases of the wave processes under study. In later phases it is probable that all these processes are modified by various types of parametric instabilities (see Bárta \& Karlický 2000). But for these studies, more extended simulations with lower noise levels are necessary.

The herringbone structures in type II solar radio bursts are the clearest evidence of beams accelerated by shocks (Mann \& Klassen 2002). But the characteristics of the herringbones indicate that these beams differ from those generating type III solar radio bursts. As in previous studies (Knocks et al. 2001, 2003; 

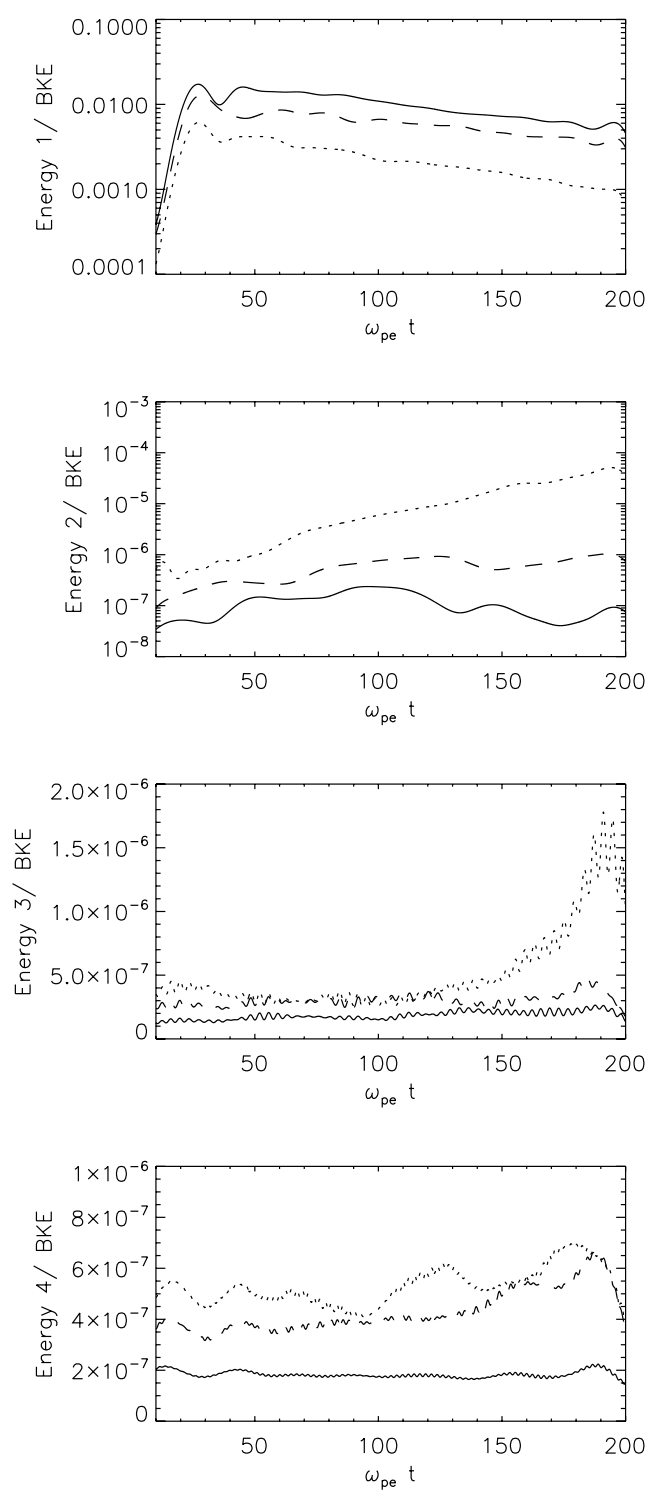

Fig. 8. Time evolution of the energy of Langmuir (Energy 1), whistler (Energy 2), left-hand (Energy 3), and right-hand (Energy 4) polarized high-frequency electromagnetic waves expressed by the ratio to the beam kinetic energy BKE in boxes 1, 2, 3, and 4 (see Figs. 6 and 7). The full line corresponds to the case with the angle $\alpha^{b}=30^{\circ}$, the dashed line to $\alpha^{b}=45^{\circ}$, and the dotted line to $\alpha^{b}=60^{\circ}$.

Mann \& Klassen 2005), we found that the beams accelerated at nearly perpendicular shocks are special beams; their distribution function has a form of the shifted loss-cone distribution that we call it the "cone" beam for short. We found that just the enhanced energy of the beam component perpendicular to the magnetic field is a source of whistler waves. For the beams generating type III radio bursts, this perpendicular beam component is probably low. Furthermore, the presence of the perpendicular component in the beam means lower propagation speed along the magnetic field lines (for the same total energy of the beam). This agrees with the lower frequency drifts of the herringbones in comparison with those of type III solar radio bursts.

Herringbones have both positive and negative frequency drifts. Therefore, to explain the herringbones by the shockreflected electrons only, we need to assume a scenario with two acceleration sites and a shock that is nearly perpendicular not only to the magnetic field but also to the density gradient
(Holman \& Pesses 1983). This model is supported by the fact that the distribution of the frequency drifts of the herringbones is more or less symmetric when comparing its parts with positive and negative frequency drifts (Mann \& Klassen 2002).

Nevertheless, it is not possible to exclude transmitted and accelerated electrons at a nearly perpendicular shock as a source for type II radio radiation and even for some herringbones. Its distribution function, which is shown in Fig. 2 (bottom part), partly resembles that for the reflected electrons (Fig. 2, upper part) and partly the single loss-cone distribution function (Moullard et al. 2001). We expect that such a distribution of the transmitted electrons can also generate Langmuir, whistler, and high-frequency electromagnetic waves similar to reflected electrons. This expectation can be supported by observations of Langmuir and whistler waves in connection with a single losscone distribution inside a magnetic cloud (Moullard et al. 2001).

Both the left and right-handed electromagnetic waves computed here can escape from the shock region and can be observed as type II radio bursts. Their ratio gives the polarization of the radio emission at the radio source. Thus, our results (Fig. 8) indicate an enhanced polarization of the radio emission for the high "cone" beam angles. But, the depolarization effects can decrease this polarization. For comparison, the polarization of the type II radio bursts is low in most cases, but there are examples with polarization up to $80 \%$ (Zlobec et al. 1993).

Acknowledgements. M.K. would like to thank Japan Society for the Promotion of Science for its invitation and support during his stay at the Toyama University. Furthermore, M.K. thanks Prof. J. I. Sakai and Dr. S. Saito for their PIC code and many useful discussions. This research was supported by Project AV0Z10030501 and by Grants IAA3003202 and 1QS300120506 of the Academy of Sciences and Grants 205/04/0358 and 205/03/0953 of Grant Agency of the Czech Republic. Authors thank the referee for useful comments.

\section{References}

Bale, S. D., Reiner, M. J., Bougeret, J. L., et al. 1999, Geophys. Res. Lett., 26, 1573

Bárta, M., \& Karlický, M. 2000, A\&A, 353, 757

Holman, G. D., \& Pesses, M. E. 1983, ApJ, 267, 837

Knock, S. A., Cairns, I. H., Robinson, P. A., \& Kuncic, Z. 2001, J. Geophys. Res., 106, 25041

Knock, S. A., Cairns, I. H., Robinson, P. A., \& Kuncic, Z. 2003, J. Geophys. Res., 108, 1126

Leroy, M., \& Mangeney, A. 1984, Ann. Geophys., 2, 449

Mann, G., \& Klassen, A. 2002, in Proc. 10th Europ. Solar Phys. Meet., ESA SP-506, 245

Mann, G., \& Klassen, A. 2005, A\&A, 441, 319

Melrose, D. B. 1980, Plasma Astrophysics, Vol. 1, Gordon and Breach Science Publ., New York, 99

Mikhailovkii, A. B. 1974, Theory of Plasma Instabilities, Vol. 1 (New York: Consultants Bureau)

Moullard, O., Burgess, D., Salem, C., et al. 2001, J. Geophys. Res., 106, 8301

Nelson, G. J., \& Melrose, D. B. 1985, in Solar Radiophysics, ed. D. J. McLean, \& N. R. Labrum (New York: Cambridge Univ. Press)

Roberts, J. A. 1959, Austr. J. Phys., 12, 322

Saito, S., \& Sakai, J. I. 2004, ApJ, 616, L179

Trávníček, P., Maksimovic, M., Cornilleau-Wehrlin, N., et al. 2002, Whistler waves observations upstream the bowshock during 31 March 2001 bow shock crossings: Cluster II observations, poster presented at 34th COSPAR Scientific Assembly, The Second World Space Congress, held 1019 October, 2002 in Houston, TX, USA

Tsytovich, V. N. 1970, Nonlinear Effects in Plasma (New York: Plenum Press)

Vandas, M. 1989a, Bull. Astron. Inst. Czech., 40, 175

Vandas, M. 1989b, Bull. Astron. Inst. Czech., 40, 189

Vandas, M., \& Karlický, M. 2000, Sol. Phys., 197, 85

Wu, C. 1984, J. Geophys. Res., 89, 8857

Zaitsev, V., Zlotnik, E., Mann, G., Aurass, H., \& Klassen, A. 1998, CESRA Workshop on Coronal Explosive Events, Metsaehovi Publications on Radio Science, HUT-MET-27, 70

Zlobec, P., Messerotti, M., Karlický, M., \& Urbarz, H. 1993, Sol. Phys., 144, 373 